PROCEEDINGS OF THE AMERICAN MATHEMATICAL SOCIETY

Volume 124, Number 1, January 1996

\title{
A PROPORTIONAL DVORETZKY-ROGERS FACTORIZATION RESULT
}

\author{
A. A. GIANNOPOULOS
}

(Communicated by Dale Alspach)

\begin{abstract}
If $X$ is an $n$-dimensional normed space and $\varepsilon \in(0,1)$, there exists $m \geq(1-\varepsilon) n$, such that the formal identity $i_{2, \infty}: l_{2}^{m} \rightarrow l_{\infty}^{m}$ can be written as $i_{2, \infty}=\alpha \circ \beta, \beta: l_{2}^{m} \rightarrow X, \alpha: X \rightarrow l_{\infty}^{m}$, with $\|\alpha\| \cdot\|\beta\| \leq c / \varepsilon$. This is proved as a consequence of a Sauer-Shelah type theorem for ellipsoids.
\end{abstract}

\section{INTRODUCTION}

A version of the classical Dvoretzky-Rogers lemma [D-R] asserts that, if $(X,\|\cdot\|)$ is an $n$-dimensional normed space, there exist vectors $x_{1}, \ldots, x_{m} \in X, m=[\sqrt{n}]$, such that for any choice of real numbers $t_{1}, \ldots, t_{m}$,

$$
\max _{j \leq m}\left|t_{j}\right| \leq\left\|\sum_{j \leq m} t_{j} x_{j}\right\|_{X} \leq c\left(\sum_{j \leq m} t_{j}^{2}\right)^{1 / 2},
$$

where $c>0$ is an absolute constant. Towards a strengthening of this result for $m$ proportional to $n$, Bourgain-Szarek [B-S] and later Szarek-Talagrand [S-T] proved the following:

Theorem 1. If $(X,\|\cdot\|)$ is an n-dimensional normed space and $\varepsilon \in(0,1)$, there exist vectors $x_{1}, \ldots, x_{m} \in X, m \geq(1-\varepsilon) n$, such that for any reals $t_{1}, \ldots, t_{m}$,

$$
\max _{j \leq m}\left|t_{j}\right| \leq\left\|\sum_{j \leq m} t_{j} x_{j}\right\|_{X} \leq c \varepsilon^{-d}\left(\sum_{j \leq m} t_{j}^{2}\right)^{1 / 2},
$$

where $c, d>0$ are absolute constants. Equivalently, the formal identity $i_{2, \infty}$ : $l_{2}^{m} \rightarrow l_{\infty}^{m}$ can be written as $i_{2, \infty}=\alpha \circ \beta$, where $\beta: l_{2}^{m} \rightarrow X, \alpha: X \rightarrow l_{\infty}^{m}$, and $\|\alpha\| \cdot\|\beta\| \leq c \varepsilon^{-d}$. The same holds true for $i_{1,2}: l_{1}^{m} \rightarrow l_{2}^{m}$.

The best possible dependence on $\varepsilon$ is not known. As shown by S. J. Szarek [Sz.1], there exists an $n$-dimensional normed space $X$ such that $\|\alpha\| \cdot\|\beta\| \geq c(n / \log n)^{1 / 10}$ whenever $i_{2, \infty}: l_{2}^{n} \rightarrow l_{\infty}^{n}$ is written as $i_{2, \infty}=\alpha \circ \beta$ ( $\alpha, \beta$ as above), and this implies that $d$ in Theorem 1 has to be at least $1 / 10$. On the other hand, in [S-T] it is proved that Theorem 1 holds with $d=2$, and in $[\mathrm{G}]$ we obtain a similar result with $d=3 / 2$. Here, we shall show that the same holds true with $d=1$.

Received by the editors February 21, 1994 and, in revised form, August 15, 1994.

1991 Mathematics Subject Classification. Primary 46B07.

(C)1996 American Mathematical Society 
Let us note that the method establishing this "proportional Dvoretzky-Rogers factorization" is closely related to the problem of the Banach-Mazur distance to the cube. A detailed exposition of the techniques used so far for both problems is given in [Sz.2].

The source of the improvement on the estimates in Theorem 1 is a Sauer-Shelah type theorem for ellipsoids, which we feel is of independent interest: The wellknown combinatorial Sauer-Shelah lemma [Sa], [Sh] states that if $0 \leq l<s$ and $M$ is a subset of $\{-1,1\}^{s}$ of cardinality $|M|>\left(\begin{array}{l}s \\ 0\end{array}\right)+\left(\begin{array}{l}s \\ 1\end{array}\right)+\cdots+\left(\begin{array}{l}s \\ l\end{array}\right)$, then there exists $\sigma \subset\{1, \ldots, s\},|\sigma|>l$, such that $P_{\sigma}(M)=\{-1,1\}^{\sigma}$, where $P_{\sigma}$ is the restriction map $\left(\delta_{j}\right)_{j \leq s} \rightarrow\left(\delta_{j}\right)_{j \in \sigma}$. A special case of this lemma is of particular interest: If $M \subset\{-1,1\}^{s}$ and $|M| \geq 2^{s-1}$, then we can find $\sigma \subset\{1, \ldots, s\},|\sigma| \geq \frac{s}{2}$, with $P_{\sigma}(M)=\{-1,1\}^{\sigma}$.

In connection with their work on the Banach-Mazur distance to the cube, Szarek and Talagrand [S-T] proved an isomorphic variant of the Sauer-Shelah lemma: If $M \subset\{-1,1\}^{s}$, viewed now as a set of points in $\mathbf{R}^{s}$, and if $|M| \geq 2^{s-1}, \varepsilon \in(0,1)$, then there exists $\sigma \subset\{1, \ldots, s\},|\sigma| \geq(1-\varepsilon) s$, such that

$$
\operatorname{absconv}\left(P_{\sigma}(M)\right) \supseteq c \varepsilon[-1,1]^{\sigma}
$$

where $c>0$ is an absolute constant (and the absolute convex hull is taken in $\mathbf{R}^{\sigma}$ ).

For our purposes, we need to consider the following situation: Let $u_{1}, \ldots, u_{s}$ be vectors in $\mathbf{R}^{n}$, of Euclidean norm $\left|u_{j}\right|_{n} \leq 1, j=1, \ldots, s$. Define the symmetric convex set

$$
\mathcal{E}=\left\{\left(\delta_{j}\right)_{j \leq s} \in \mathbf{R}^{s}:\left|\sum_{j \leq s} \delta_{j} u_{j}\right|_{n} \leq 1\right\} .
$$

(Note that if $s \leq n$ and the vectors $u_{j}$ are linearly independent in $\mathbf{R}^{n}$, then $\mathcal{E}$ is an ellipsoid in $\mathbf{R}^{s}$. This will be the context in the proof of Theorem 1.) Again, we are interested in the "size" of the image $P_{\sigma}(\mathcal{E})$ of $\mathcal{E}$ for "large" subsets $\sigma$ of $\{1, \ldots, s\}$. Our main result is then the following

Theorem 2. If $u_{j} \in \mathbf{R}^{n},\left|u_{j}\right|_{n} \leq 1, j=1, \ldots, s$, and

$$
\mathcal{E}=\left\{\left(\delta_{j}\right)_{j \leq s} \in \mathbf{R}^{s}:\left|\sum_{j \leq s} \delta_{j} u_{j}\right|_{n} \leq 1\right\},
$$

then for every $\varepsilon \in(0,1)$, we can find $\sigma \subseteq\{1, \ldots, s\},|\sigma| \geq(1-\varepsilon)$, such that

$$
P_{\sigma}(\mathcal{E}) \supseteq c \sqrt{\varepsilon} D_{\sigma},
$$

where $D_{\sigma}$ is the Euclidean unit ball in $\mathbf{R}^{\sigma}$ and $c>0$ is an absolute constant.

We shall use the standard notation from $[\mathrm{M}-\mathrm{Sc}]$ or $[\mathrm{T}-\mathrm{J}]$. By $|\cdot|$ we denote the cardinality of a finite set. The letter $c$ will always denote an absolute positive constant, not necessarily the same in all its occurrences. For basic facts about $p$ absolutely summing operators, used in the proof of Theorem 2 , we refer the reader to $[\mathrm{L}-\mathrm{T}],[\mathrm{Pi}]$, and $[\mathrm{T}-\mathrm{J}]$.

\section{Proof of Theorem 2}

First, we introduce some additional notation: The set $S=\{1, \ldots, s\}$, as well as $\mathbf{R}^{s}$, will be fixed throughout the proof. If $\varphi \subseteq S$, then $\mathbf{R}^{\varphi}=\left\{\left(\delta_{j}\right)_{j \leq s} \in \mathbf{R}^{s}: \delta_{j}=0\right.$ if $j \notin \varphi\}$. A point in $\mathbf{R}^{s}$ denoted by $\left(\delta_{j}\right)_{j \in \varphi}$ is assumed to satisfy $\delta_{j}=0$ if $j \notin \varphi$. 
If $\tau, \varphi$ are disjoint subsets of $S$ and $A \subseteq \mathbf{R}^{\varphi}$, we sometimes write $\mathbf{0}_{\tau} \times A$ instead of $A$ to indicate that $A$ is to be understood as a subset of $\mathbf{R}^{\varphi \cup \tau}$. In particular, if $\varphi \subseteq S_{1} \subseteq S$ and $L>0$, then

$$
\begin{aligned}
I_{L, \varphi, S_{1}} & =\mathbf{0}_{\varphi} \times\{-L, L\}^{S_{1} \backslash \varphi} \\
& =\left\{\left(\delta_{j}\right)_{j \in S_{1}} \in \mathbf{R}^{S_{1}}: \delta_{j}=\left\{\begin{array}{ll}
0 & \text { if } j \in \varphi, \\
\pm L & \text { if } j \in S_{1} \backslash \varphi
\end{array}\right\} .\right.
\end{aligned}
$$

Note that $\left|I_{L, \varphi, S_{1}}\right|=2^{\left|S_{1} \backslash \varphi\right|}$. If $a \in \mathbf{R}^{\varphi}, b \in \mathbf{R}^{\tau}$, and $\tau, \varphi$ are disjoint subsets of $S$, then $(a, b) \in \mathbf{R}^{\varphi \cup \tau}$ is the sum $a+b$. Finally, if $S_{1}$ is a non-empty subset of $S$, we define

$$
\mathcal{E}_{S_{1}}=\left\{\left(\delta_{j}\right)_{j \in S_{1}} \in \mathbf{R}^{S_{1}}:\left|\sum_{j \in S_{1}} \delta_{j} u_{j}\right|_{n} \leq 1\right\} .
$$

Our starting point is then an immediate consequence of the Sauer-Shelah lemma:

Lemma 1. If $L>0, \varphi \subseteq S_{1} \subseteq S$, and $M \subseteq \mathbf{0}_{\varphi} \times\{-L, L\}^{S_{1} \backslash \varphi}$, with $|M| \geq$ $2^{\left|S_{1} \backslash \varphi\right|-1}$, then there exists $\sigma \subseteq S_{1} \backslash \varphi,|\sigma| \geq \frac{\left|S_{1} \backslash \varphi\right|}{2}$, such that

$$
P_{\varphi \cup \sigma}(M)=\mathbf{0}_{\varphi} \times\{-L, L\}^{\sigma} .
$$

Using an inductive argument based on Lemma 1, we obtain a first result on the size of the projections of $\mathcal{E}_{S_{1}}$, for an arbitrary $S_{1} \subseteq S$. This step is crucial for our proof of Theorem 2, so we state it as our next lemma and give its proof, although it can essentially be found in $[\mathrm{G}]$.

Lemma 2. If $\emptyset \neq S_{1} \subseteq S$ and $\varepsilon \in(0,1)$ are given, then there exists $\sigma \subseteq S_{1}$, with $|\sigma| \geq(1-\varepsilon)\left|S_{1}\right|$, such that

$$
P_{\sigma}\left(\mathcal{E}_{S_{1}}\right) \supseteq \frac{c \sqrt{\varepsilon}}{\sqrt{\left|S_{1}\right|}}[-1,1]^{\sigma}
$$

where $c>0$ is an absolute constant.

Proof. Set $\alpha_{k}=\sum_{r=0}^{k-1} 2^{r / 2}, \beta_{k}=\sum_{r=0}^{k-1} 2^{r}=2^{k}-1$, and $Q_{\tau}=[-1,1]^{\tau}$ for every non-empty $\tau \subseteq S_{1}$.

We shall prove by induction that:

$$
\text { For } k=1,2, \ldots \text {, one can find } \sigma_{k} \subseteq S_{1} \text {, with }\left|\sigma_{k}\right| \geq
$$

$$
\begin{aligned}
& \left(1-\frac{1}{2^{k}}\right)\left|S_{1}\right|, \text { such that } \\
& Q_{\sigma_{k}} \subseteq P_{\sigma_{k}}\left(\alpha_{k} \sqrt{2\left|S_{1}\right|} \mathcal{E}_{S_{1}} \cap \beta_{k} Q_{S_{1}}\right) .
\end{aligned}
$$

Since $\alpha_{k} \leq \frac{2^{k / 2}}{\sqrt{2}-1}$, condition ( $*$ ) clearly implies that, for $k=1,2, \ldots$,

$$
P_{\sigma_{k}}\left(\mathcal{E}_{S_{1}}\right) \supseteq \frac{c}{\sqrt{\left|S_{1}\right|}} \sqrt{\frac{1}{2^{k}}}[-1,1]^{\sigma_{k}}
$$

with $c=1-\frac{1}{\sqrt{2}}$, which is the assertion of the lemma for $\varepsilon=1 / 2^{k}$. The continuous version will easily follow with a worse constant $c$.

Inductive step. Consider the set $J_{k}=\mathbf{0}_{\sigma_{k}} \times\left\{-2^{k / 2}, 2^{k / 2}\right\} S_{1} \backslash \sigma_{k}$, where $\sigma_{k}$ is the subset of $S_{1}$ given by $(*)$. Note that $\left|J_{k}\right|=2^{\left|S_{1} \backslash \sigma_{k}\right|}$. By the parallelogram law and 
the fact that $\left|S_{1} \backslash \sigma_{k}\right| \leq\left|S_{1}\right| / 2^{k}$, we have

$$
\underset{\left(\delta_{j}\right) \in J_{k}}{\operatorname{Ave}}\left|\sum_{j \in S_{1}} \delta_{j} u_{j}\right|_{n}^{2}=2^{k} \sum_{j \in S_{1} \backslash \sigma_{k}}\left|u_{j}\right|_{n}^{2} \leq\left|S_{1}\right|,
$$

and Markov's inequality implies that there exists $M^{k+1} \subseteq J_{k} \cap \sqrt{2\left|S_{1}\right|} \mathcal{E}_{S_{1}}$ with $\left|M^{k+1}\right| \geq 2^{\left|S_{1} \backslash \sigma_{k}\right|-1}$. Then, by Lemma 1 , we can find $\sigma_{k+1}^{*} \subseteq S_{1} \backslash \sigma_{k}$, of cardinality $\left|\sigma_{k+1}^{*}\right| \geq \frac{\left|S_{1} \backslash \sigma_{k}\right|}{2}$, for which

$$
P_{\sigma_{k} \cup \sigma_{k+1}^{*}}\left(M^{k+1}\right)=\mathbf{0}_{\sigma_{k}} \times\left\{-2^{k / 2}, 2^{k / 2}\right\}^{\sigma_{k+1}^{*}} .
$$

Since $M^{k+1} \subseteq \sqrt{2\left|S_{1}\right|} \mathcal{E}_{S_{1}} \cap 2^{k / 2} Q_{S_{1}}$, it follows that

$$
\mathbf{0}_{\sigma_{k}} \times 2^{k} Q_{\sigma_{k+1}^{*}} \subseteq P_{\sigma_{k} \cup \sigma_{k+1}^{*}}\left(2^{k / 2} \sqrt{2\left|S_{1}\right|} \mathcal{E}_{S_{1}} \cap 2^{k} Q_{S_{1}}\right) .
$$

Suppose now that $a \in Q_{\sigma_{k}}, b \in Q_{\sigma_{k+1}^{*}}$. From the inductive hypothesis $(*)$, there exists $t_{a} \in \alpha_{k} \sqrt{2\left|S_{1}\right|} \mathcal{E}_{S_{1}} \cap \beta_{k} Q_{S_{1}}$ such that $P_{\sigma_{k}}\left(t_{a}\right)=a$. Let $w_{a}=P_{\sigma_{k+1}^{*}}\left(t_{a}\right)$; then $w_{a} \in \beta_{k} Q_{\sigma_{k+1}^{*}}$ and

$$
\left(a, w_{a}\right) \in P_{\sigma_{k} \cup \sigma_{k+1}^{*}}\left(\alpha_{k} \sqrt{2\left|S_{1}\right|} \mathcal{E}_{S_{1}} \cap \beta_{k} Q_{S_{1}}\right) .
$$

If $v_{a, b}=b-w_{a}$, it is clear that $v_{a, b} \in Q_{\sigma_{k+1}^{*}}+\beta_{k} Q_{\sigma_{k+1}^{*}}=2^{k} Q_{\sigma_{k+1}^{*}}$, and therefore, by $(* *)$,

$$
\left(\mathbf{0}_{\sigma_{k}}, v_{a, b}\right) \in P_{\sigma_{k} \cup \sigma_{k+1}^{*}}\left(2^{k / 2} \sqrt{2\left|S_{1}\right|} \mathcal{E}_{S_{1}} \cap 2^{k} Q_{S_{1}}\right) .
$$

Then,

$$
\begin{aligned}
(a, b)= & \left(a, w_{a}\right)+\left(\mathbf{0}_{\sigma_{k}}, v_{a, b}\right) \in P_{\sigma_{k} \cup \sigma_{k+1}^{*}}\left(\alpha_{k} \sqrt{2\left|S_{1}\right|} \mathcal{E}_{S_{1}} \cap \beta_{k} Q_{S_{1}}\right) \\
& +P_{\sigma_{k} \cup \sigma_{k+1}^{*}}\left(2^{k / 2} \sqrt{2\left|S_{1}\right|} \mathcal{E}_{S_{1}} \cap 2^{k} Q_{S_{1}}\right) \\
\subseteq & P_{\sigma_{k} \cup \sigma_{k+1}^{*}}\left(\alpha_{k+1} \sqrt{2\left|S_{1}\right|} \mathcal{E}_{S_{1}} \cap \beta_{k+1} Q_{S_{1}}\right) .
\end{aligned}
$$

Since $a \in Q_{\sigma_{k}}, b \in Q_{\sigma_{k+1}^{*}}$ were arbitrary, this means that

$$
Q_{\sigma_{k} \cup \sigma_{k+1}^{*}} \subseteq P_{\sigma_{k} \cup \sigma_{k+1}^{*}}\left(\alpha_{k+1} \sqrt{2\left|S_{1}\right|} \mathcal{E}_{S_{1}} \cap \beta_{k+1} Q_{S_{1}}\right) .
$$

If we define $\sigma_{k+1}=\sigma_{k} \cup \sigma_{k+1}^{*}$, we readily see that $\left|\sigma_{k+1}\right| \geq\left(1-\frac{1}{2^{k+1}}\right)\left|S_{1}\right|$, and this completes the inductive step. The first step $(k=1)$ is much simpler.

For our next two lemmas we shall need to assume that the vectors $u_{1}, \ldots, u_{s}$ are linearly independent.

Lemma 3. Let $S_{1}$ be a non-empty subset of $S$. Then, for every $\theta \in\left(0, \frac{1}{4}\right)$, we can find disjoint $\sigma, \tau \subseteq S_{1}$ with $|\sigma| \geq \frac{\left|S_{1}\right|}{2},|\tau| \leq \theta\left|S_{1}\right|$, and

$$
P_{S_{1} \backslash \tau}\left(\mathcal{E}_{S_{1}}\right) \supseteq \mathbf{0}_{S_{1} \backslash(\sigma \cup \tau)} \times c \sqrt{\theta} D_{\sigma},
$$

where $c>0$ is an absolute constant. 
Proof. Set $V_{S_{1}}=\operatorname{span}\left\{u_{j}, j \in S_{1}\right\}$. Then, there exist $x_{i} \in V_{S_{1}}, i \in S_{1}$, such that

$$
\left\langle x_{i}, u_{j}\right\rangle=\delta_{i j} \quad \text { for any pair of } i, j \in S_{1} .
$$

Applying Lemma 2 for the ellipsoid $\mathcal{E}_{S_{1}}$, we obtain $\tau \subseteq S_{1},|\tau| \leq \theta\left|S_{1}\right|$, for which

$$
P_{S_{1} \backslash \tau}\left(\mathcal{E}_{S_{1}}\right) \supseteq \frac{c \sqrt{\theta}}{\sqrt{\left|S_{1}\right|}}[-1,1]^{S_{1} \backslash \tau} .
$$

Then, for any choice of scalars $\left(t_{i}\right)_{i \in S_{1} \backslash \tau}$, we can find a vector $\left(\delta_{j}\right)_{j \in S_{1}}$ in $\mathcal{E}_{S_{1}}$ whose restriction in $\mathbf{R}^{S_{1} \backslash \tau}$ is $\left(\frac{c \sqrt{\theta}}{\sqrt{\left|S_{1}\right|}} \operatorname{sign} t_{j}\right)_{j \in S_{1} \backslash \tau}$. In view of the orthogonality relations between the $x_{i}$ 's and the $u_{j}$ 's we see that

$$
\begin{aligned}
\sum_{i \in S_{1} \backslash \tau}\left|t_{i}\right| & =\left\langle\sum_{i \in S_{1} \backslash \tau} t_{i} x_{i}, \sum_{j \in S_{1} \backslash \tau}\left(\operatorname{sign} t_{j}\right) u_{j}\right\rangle \\
& =\frac{\sqrt{\left|S_{1}\right|}}{c \sqrt{\theta}}\left\langle\sum_{i \in S_{1} \backslash \tau} t_{i} x_{i}, \sum_{j \in S_{1}} \delta_{j} u_{j}\right\rangle \\
& \leq \frac{\sqrt{\left|S_{1}\right|}}{c \sqrt{\theta}}\left|\sum_{i \in S_{1} \backslash \tau} t_{i} x_{i}\right|_{n}\left|\sum_{j \in S_{1}} \delta_{j} u_{j}\right|_{n} \\
& \leq \frac{\sqrt{\left|S_{1}\right|}}{c \sqrt{\theta}}\left|\sum_{i \in S_{1} \backslash \tau} t_{i} x_{i}\right|_{n} .
\end{aligned}
$$

It follows that the operator $T: \operatorname{span}\left\{x_{i}, i \in S_{1} \backslash \tau\right\} \subset l_{2}^{n} \rightarrow l_{1}^{\left|S_{1} \backslash \tau\right|}$, defined by $T x_{i}=e_{i}$ (where $\left\{e_{i}\right\}$ is the canonical orthonormal basis in $\mathbf{R}^{\left|S_{1} \backslash \tau\right|}$ ), has norm not exceeding $\sqrt{\left|S_{1}\right|} / c \sqrt{\theta}$. Then, $T^{*}: l_{\infty}^{\left|S_{1} \backslash \tau\right|} \rightarrow l_{2}^{n}$ is a 2-absolutely summing operator with 2-summing norm $\pi_{2}\left(T^{*}\right) \leq K_{G} \frac{\sqrt{\left|S_{1}\right|}}{c \sqrt{\theta}}$, where $K_{G}$ is Grothendieck's constant. From Pietch's factorization theorem, applied in the same context as in the proof of Theorem 1.2 [B-T], we can find positive real numbers $\lambda_{i}, i \in S_{1} \backslash \tau$, with $\sum_{i \in S_{1} \backslash \tau} \lambda_{i}^{2}=1$, such that, for any reals $t_{i}, i \in S_{1} \backslash \tau$,

$$
\left(\sum_{i \in S_{1} \backslash \tau}\left(\frac{t_{i}}{\lambda_{i}}\right)^{2}\right)^{1 / 2} \leq K_{G} \frac{\sqrt{\left|S_{1}\right|}}{c \sqrt{\theta}}\left|\sum_{i \in S_{1} \backslash \tau} t_{i} x_{i}\right|_{n} .
$$

Since $\sum_{i \in S_{1} \backslash \tau} \lambda_{i}^{2}=1$ and $\theta<\frac{1}{4}$, we apply Markov's inequality to obtain $\sigma \subseteq$ $S_{1} \backslash \tau,|\sigma| \geq \frac{\left|S_{1}\right|}{2}$, with $\lambda_{i} \leq \frac{2}{\sqrt{\left|S_{1}\right|}}$ for every $i \in \sigma$. Suppose now that $\left(\delta_{j}\right)_{j \in \sigma} \in D_{\sigma}$ is given, i.e. $\sum_{j \in \sigma} \delta_{j}^{2} \leq 1$. The set $\left\{u_{j}, j \in \tau\right\} \cup\left\{x_{i}, i \in S_{1} \backslash \tau\right\}$ is linearly independent (hence a basis) in $V_{S_{1}}$, so we can write

$$
\sum_{j \in \sigma} \delta_{j} u_{j}+\sum_{j \in \tau} \rho_{j} u_{j}=\sum_{i \in S_{1} \backslash \tau} t_{i} x_{i}
$$


for suitable $\left(\rho_{j}\right)_{j \in \tau},\left(t_{i}\right)_{i \in S_{1} \backslash \tau}$. Then,

$$
\begin{aligned}
\left|\sum_{j \in \sigma} \delta_{j} u_{j}+\sum_{j \in \tau} \rho_{j} u_{j}\right|_{n}^{2} & =\left\langle\sum_{j \in \sigma} \delta_{j} u_{j}+\sum_{j \in \tau} \rho_{j} u_{j}, \sum_{i \in S_{1} \backslash \tau} t_{i} x_{i}\right\rangle \\
& =\left\langle\sum_{j \in \sigma} \delta_{j} u_{j}, \sum_{i \in \sigma} t_{i} x_{i}\right\rangle=\sum_{i \in \sigma} \delta_{i} t_{i} \leq\left(\sum_{i \in \sigma} t_{i}^{2}\right)^{1 / 2} \\
& \leq\left(\sum_{i \in \sigma}\left(\frac{t_{i}}{\lambda_{i}}\right)^{2}\right)^{1 / 2} \frac{2}{\sqrt{\left|S_{1}\right|}} \leq\left(\sum_{i \in S_{1} \backslash \tau}\left(\frac{t_{i}}{\lambda_{i}}\right)^{2}\right)^{1 / 2} \frac{2}{\sqrt{\left|S_{1}\right|}} \\
& \leq\left.\frac{2 K_{G}}{c} \frac{1}{\sqrt{\theta}} \sum_{i \in S_{1} \backslash \tau} t_{i} x_{i}\right|_{n}
\end{aligned}
$$

and therefore,

$$
\left|\sum_{j \in \sigma} \delta_{j} u_{j}+\sum_{j \in \tau} \rho_{j} u_{j}\right|_{n} \leq \frac{2 K_{G}}{c} \frac{1}{\sqrt{\theta}} .
$$

This means that $\mathbf{0}_{S_{1} \backslash(\sigma \cup \tau)} \times\left(\delta_{j}\right)_{j \in \sigma} \in \frac{1}{c_{1} \sqrt{\theta}} P_{S_{1} \backslash \tau}\left(\mathcal{E}_{S_{1}}\right)$ with $c_{1}=c / 2 K_{G}$. Since $\left(\delta_{j}\right)_{j \in \sigma}$ was arbitrary in $D_{\sigma}$, the lemma follows.

We are now ready to prove Theorem 2 in the case of independent $u_{j}$ 's:

Lemma 4. For every $\varepsilon \in(0,1)$ one can find $\sigma \subseteq S,|\sigma| \geq(1-\varepsilon)$ s, such that

$$
P_{\sigma}(\mathcal{E}) \supseteq c \sqrt{\varepsilon} D_{\sigma},
$$

where $c>0$ is an absolute constant.

Proof. Given $\varepsilon \in(0,1)$, we set $\theta=\varepsilon / 7$. Let also $k$ be the non-negative integer for which $\frac{1}{2^{k+1}} \leq \varepsilon<\frac{1}{2^{k}}$. To obtain $\sigma$, we shall follow an inductive procedure based on Lemma 3:

Step 1: We set $S_{0}=S$, and $\theta_{1}=\theta$. Since $\theta_{1} \in\left(0, \frac{1}{4}\right)$, we can find a pair $\left(\sigma_{1}, \tau_{1}\right)$ of disjoint subsets of $S_{0}$, with $\left|\tau_{1}\right| \leq \theta_{1}\left|S_{0}\right|,\left|\sigma_{1}\right| \geq \frac{1}{2}\left|S_{0}\right|$, and $P_{S_{0} \backslash \tau_{1}}\left(\mathcal{E}_{S_{0}}\right) \supseteq$ $\mathbf{0}_{S_{0} \backslash\left(\sigma_{1} \cup \tau_{1}\right)} \times c \sqrt{\theta_{1}} D_{\sigma_{1}}$, where $c$ is the constant from Lemma 3. Finally, we define $S_{1}=S_{0} \backslash\left(\sigma_{1} \cup \tau_{1}\right)$. Note that $\left|S_{1}\right| \leq \frac{1}{2}\left|S_{0}\right|=\frac{s}{2}$.

Inductive step: Suppose that $S_{l}$ has been defined, and $\left|S_{l}\right|>\frac{\varepsilon}{2} s$. If, in addition, $l<k+2$, we define $\theta_{l+1}=2^{l / 2} \theta$. Note that then $\theta_{l+1} \leq \frac{\sqrt{2}}{7} 2^{k / 2} \varepsilon<$ $\frac{\sqrt{2}}{7} \sqrt{\varepsilon}<\frac{1}{4}$, and therefore we can apply Lemma 3 for $\mathcal{E}_{S_{l}}$ and $\theta_{l+1}$ to obtain a pair $\left(\sigma_{l+1}, \tau_{l+1}\right)$ of disjoint subsets of $S_{l}$, with $\left|\tau_{l+1}\right| \leq \theta_{l+1}\left|S_{l}\right|,\left|\sigma_{l+1}\right| \geq \frac{1}{2}\left|S_{l}\right|$, and $P_{S_{l} \backslash \tau_{l+1}}\left(\mathcal{E}_{S_{l}}\right) \supseteq \mathbf{0}_{S_{l} \backslash\left(\sigma_{l+1} \cup \tau_{l+1}\right)} \times c \sqrt{\theta_{l+1}} D_{\sigma_{l+1}}$. To complete the inductive step, we define $S_{l+1}=S_{l} \backslash\left(\sigma_{l+1} \cup \tau_{l+1}\right)$. Note also that $\left|S_{l+1}\right| \leq \frac{1}{2}\left|S_{l}\right|$, hence, as far as we continue performing these steps, $\left|S_{l}\right| \leq \frac{s}{2^{l}}$.

We end this inductive construction when we arrive at a set $S_{l}$ of cardinality $\left|S_{l}\right| \leq \frac{\varepsilon}{2} s$. This will certainly happen after at most $(k+2)$-steps, since $\frac{1}{2^{k+2}} \leq \frac{\varepsilon}{2}$ and our construction implies that $\left|S_{l}\right|<\frac{s}{2^{l}}$ for every admissible $l$.

Suppose $l_{*}$ is the first index for which $\left|S_{l_{*}}\right| \leq \frac{\varepsilon}{2} s$. We define $\sigma=\sigma_{1} \cup \cdots \cup \sigma_{l_{*}}$. 
Claim 1. $|\sigma| \geq(1-\varepsilon) s$.

[Proof. Note that $\bigcup_{1 \leq l \leq l_{*}}\left(\sigma_{l} \cup \tau_{l}\right)=S \backslash S_{l_{*}}$, hence

$$
\begin{aligned}
|S \backslash \sigma| & =\left|S_{l_{*}}\right|+\sum_{1 \leq l \leq l_{*}}\left|\tau_{l}\right| \leq \frac{\varepsilon}{2} s+\sum_{1 \leq l \leq l_{*}} \theta_{l}\left|S_{l-1}\right| \\
& \left.\leq \frac{\varepsilon}{2} s+\theta \sum_{1 \leq l \leq l_{*}} 2^{(l-1) / 2} \frac{s}{2^{l-1}}<\frac{\varepsilon}{2} s+\frac{\varepsilon}{7} s\left(\sum_{l=0}^{\infty} \frac{1}{2^{l / 2}}\right)<\varepsilon s .\right]
\end{aligned}
$$

Claim 2. If $1 \leq l \leq l_{*}$, then

$$
P_{\sigma}(\mathcal{E}) \supseteq \mathbf{0}_{\sigma \backslash \sigma_{l}} \times c 2^{(l-1) / 4} \sqrt{\theta} D_{\sigma_{l}} .
$$

[Proof. Suppose that $\Delta_{l}=\left(\delta_{j}\right)_{j \in \sigma_{l}} \in c 2^{(l-1) / 4} \sqrt{\theta} D_{\sigma_{l}}$. Then our construction implies that $\mathbf{0}_{S_{l-1} \backslash\left(\sigma_{l} \cup \tau_{l}\right)} \times \Delta_{l} \in P_{S_{l-1} \backslash \tau_{l}}\left(\mathcal{E}_{S_{l-1}}\right)$. Hence, we can find $\left(\zeta_{i}\right)_{i \in \tau_{l}}$ such that

$$
\left|\sum_{j \in \sigma_{l}} \delta_{j} u_{j}+\sum_{i \in \tau_{l}} \zeta_{i} u_{i}\right|_{n} \leq 1 .
$$

Since $\sigma \cap \tau_{l}=\emptyset$, it is clear that $\mathbf{0}_{\sigma \backslash \sigma_{l}} \times \Delta_{l} \in P_{\sigma}(\mathcal{E})$.]

To conclude the proof of the lemma, suppose that $\Delta=\left(\delta_{j}\right)_{j \in \sigma}$ is an arbitrary point in $D_{\sigma}$, i.e. $\sum_{j \in \sigma} \delta_{j}^{2} \leq 1$. Consider the restriction $\Delta_{l}=\mathbf{0}_{\sigma \backslash \sigma_{l}} \times\left(\delta_{j}\right)_{j \in \sigma_{l}}$ of $\Delta$ in $\mathbf{R}^{\sigma_{l}}$, and set $\left|\Delta_{l}\right|=\left(\sum_{j \in \sigma_{l}} \delta_{j}^{2}\right)^{1 / 2}, 1 \leq l<l_{*}$. By Claim 2, each $\Delta_{l}$ belongs to $\frac{\left|\Delta_{l}\right|}{c 2^{(l-1) / 4} \sqrt{\theta}} P_{\sigma}(\mathcal{E}) ;$ thus

$$
\begin{aligned}
\Delta & =\sum_{1 \leq l \leq l_{*}} \Delta_{l} \in\left(\sum_{1 \leq l \leq l_{*}} \frac{\left|\Delta_{l}\right|}{c 2^{(l-1) / 4} \sqrt{\theta}}\right) P_{\sigma}(\mathcal{E}) \\
& \subseteq \frac{1}{c \sqrt{\theta}}\left(\sum_{1 \leq l \leq l_{*}}\left|\Delta_{l}\right|^{2}\right)^{1 / 2}\left(\sum_{l=0}^{\infty} \frac{1}{2^{l / 2}}\right)^{1 / 2} P_{\sigma}(\mathcal{E}) \\
& \subseteq \frac{\sqrt{7}}{c}\left(\frac{\sqrt{2}}{\sqrt{2}-1}\right)^{1 / 2} \frac{1}{\sqrt{\varepsilon}} P_{\sigma}(\mathcal{E}),
\end{aligned}
$$

and the lemma is proved with $c^{\prime}=c / 5$.

Proof of Theorem 2. Assume that $u_{1}, \ldots, u_{s}$ are arbitrary vectors in $\mathbf{R}^{n}$ with $\left|u_{j}\right|_{n}$ $\leq 1, j=1, \ldots, s$. Set $v_{j}=u_{j}+e_{j+n}, j=1, \ldots, s$, where $\left\{e_{i}\right\}_{i \leq n+s}$ is the canonical orthonormal basis in $\mathbf{R}^{n+s}$. Then, the $v_{j}$ 's are linearly independent vectors in $\mathbf{R}^{n+s}$, of Euclidean norm at most $\sqrt{2}$, and if

$$
\mathcal{E}^{*}=\left\{\left(\delta_{j}\right)_{j \leq s}:\left|\sum_{j \leq s} \delta_{j} v_{j}\right|_{n+s} \leq 1\right\},
$$

Lemma 4 implies that, given $\varepsilon \in(0,1)$, there exists $\sigma \subseteq S,|\sigma| \geq(1-\varepsilon) s$, for which

$$
P_{\sigma}\left(\mathcal{E}^{*}\right) \supseteq c^{\prime \prime} \sqrt{\varepsilon} D_{\sigma}
$$

with $c^{\prime \prime}=c / \sqrt{2}, c$ the constant from Lemma 4 . Since

$$
\left|\sum_{j \leq s} \delta_{j} v_{j}\right|_{n+s}^{2}=\left|\sum_{j \leq s} \delta_{j} u_{j}\right|_{n}^{2}+\sum_{j \leq s} \delta_{j}^{2}
$$


we readily see that

$$
P_{\sigma}(\mathcal{E}) \supseteq c^{\prime \prime} \sqrt{\varepsilon} D_{\sigma}
$$

and Theorem 2 is proved.

\section{Proof of Theorem 1 with $d=1$}

For the proof of the proportional Dvoretzky-Rogers factorization result, we shall combine Theorem 2 with the method used in [S-T]: Let $X=\left(\mathbf{R}^{n},\|\cdot\|\right)$ be an $n$-dimensional normed space and $\varepsilon \in(0,1)$ be given. Without loss of generality, we may assume that the ellipsoid of minimal volume containing the unit ball $B_{X}$ of $X$ is the Euclidean unit ball $D$. By John's theorem [J], $D \subseteq \sqrt{n} B_{X}$. We can also find contact points $y_{i}, i \leq N,\left\|y_{i}\right\|_{X}=\left|y_{i}\right|_{n}=1, N=O\left(n^{2}\right)$, and positive real numbers $\mu_{i}, i \leq N$, such that the following representation of the identity holds: for every $x \in \mathbf{R}^{n}, x=\sum_{i \leq N} \mu_{i}\left\langle x, y_{i}\right\rangle y_{i}$. Now, if $s$ is the smallest integer $\geq\left(1-\frac{\varepsilon}{2}\right) n$, we can choose $x_{1}, \ldots, x_{s}$ among the $y_{i}$ 's so that:

Lemma $5([\mathrm{~S}-\mathrm{T}]) \cdot \operatorname{dist}\left(x_{i}, \operatorname{span}\left\{x_{j}, j \neq i\right\}\right) \geq \sqrt{\frac{\varepsilon}{2}}, i=1, \ldots, s$.

Hence, there exist $v_{j}, j \leq s$, in $\operatorname{span}\left\{x_{i}, i \leq s\right\}$ satisfying

(i) $\left|v_{j}\right|_{n} \leq \sqrt{2 / \varepsilon}, j=1, \ldots, s$,

(ii) $\left\langle x_{i}, v_{j}\right\rangle=\delta_{i j}, i, j=1, \ldots, s$.

Set $u_{j}=\sqrt{\varepsilon / 2} v_{j}$ and define $\mathcal{E}=\left\{\left(\delta_{j}\right)_{j \leq s}:\left|\sum_{j \leq s} \delta_{j} u_{j}\right|_{n} \leq 1\right\}$. From Theorem 2 we obtain $\sigma \subseteq S,|\sigma| \geq\left(1-\frac{\varepsilon}{2}\right) s$, with $P_{\sigma}(\mathcal{E}) \supseteq c \sqrt{\varepsilon} D_{\sigma}$. Then $|\sigma| \geq(1-\varepsilon) n$, and for any choice of scalars $\mathbf{t}=\left(t_{i}\right)_{i \in \sigma}$ we have

$$
|\mathbf{t}|^{2}=\sum_{i \in \sigma} t_{i}^{2}=\left\langle\sum_{i \in \sigma} t_{i} x_{i}, \sum_{j \in \sigma} t_{j} v_{j}\right\rangle=\sqrt{\frac{2}{\varepsilon}}\left\langle\sum_{i \in \sigma} t_{i} x_{i}, \sum_{j \in \sigma} t_{j} u_{j}\right\rangle .
$$

We can extend $\left(\frac{c \sqrt{\varepsilon}}{|\mathbf{t}|} t_{j}\right)_{j \in \sigma}$ to a vector $\left(\delta_{j}\right)_{j \leq s}$ in $\mathcal{E}$. Hence,

$$
|\mathbf{t}|^{2}=\sqrt{\frac{2}{\varepsilon}} \frac{|\mathbf{t}|}{c \sqrt{\varepsilon}}\left\langle\sum_{i \in \sigma} t_{i} x_{i}, \sum_{j \leq s} \delta_{j} u_{j}\right\rangle \leq \frac{c^{\prime}}{\varepsilon}|\mathbf{t}|\left|\sum_{i \in \sigma} t_{i} x_{i}\right|_{n}
$$

and, since $|\cdot|_{n} \leq\|\cdot\|_{X}$ and the $x_{i}$ 's are of $\|\cdot\|$-norm one, we have

$$
\left(\sum_{i \in \sigma} t_{i}^{2}\right)^{1 / 2} \leq \frac{c^{\prime}}{\varepsilon}\left|\sum_{i \in \sigma} t_{i} x_{i}\right|_{n} \leq \frac{c^{\prime}}{\varepsilon}\left\|\sum_{i \in \sigma} t_{i} x_{i}\right\|_{X} \leq \frac{c^{\prime}}{\varepsilon} \sum_{i \in \sigma}\left|t_{i}\right| .
$$

Defining $\beta: l_{1}^{|\sigma|} \rightarrow X$ with $\beta\left(e_{i}\right)=x_{i}, i \in \sigma$, and $\alpha: X \rightarrow l_{2}^{|\sigma|}$ with $\alpha=T P_{\sigma}$ where $P_{\sigma}$ is the orthogonal projection of $X$ onto $\operatorname{span}\left\{x_{i}, i \in \sigma\right\}$ and $T x_{i}=e_{i}$, we have a factorization $i_{1,2}=\alpha \circ \beta$ of the identity $i_{1,2}: l_{1}^{|\sigma|} \rightarrow l_{2}^{|\sigma|}$ with $\|\alpha\| \cdot\|\beta\| \leq c^{\prime} / \varepsilon$. By duality and by the extension property of $l_{\infty}^{n}$, this is then equivalent to the assertion of the theorem.

\section{ACKNOWLEDGMENT}

This work was done while the author was visiting Case Western Reserve University. I would like to thank the Department of Mathematics for the hospitality and Professor S. J. Szarek for helpful discussions. 


\section{REFERENCES}

[B-S] J. Bourgain and S. J. Szarek, The Banach-Mazur distance to the cube and the DvoretzkyRogers factorization, Israel J. Math. 62 1988, 169-180. MR 89g:46026

[B-T] J. Bourgain and L. Tzafriri, Invertibility of "large" submatrices with applications to the geometry of Banach spaces and harmonic analysis, Israel J. Math. 57 (1987), 137-224. MR 89a: 46035

[D-R] A. Dvoretzky and C. A. Rogers, Absolute and unconditional convergence in normed linear spaces, Proc. Nat. Acad. Sci. U.S.A. 36 (1950), 192-197. MR 11:525a

[G] A. A. Giannopoulos, A note on the Banach-Mazur distance to the cube, GAFA Seminar (to appear).

$[\mathrm{J}] \quad$ F. John, Extremum problems with inequalities as subsidiary conditions, Courant Anniversary Volume, Interscience, New York, 1948. MR 10:719b

[L-T] J. Lindenstrauss and L. Tzafriri, Classical Banach spaces I: Sequence spaces, SpringerVerlag, Berlin and New York, 1977. MR 58:17766

[M-Sc] V. D. Milman and G. Schechtman, Asymptotic theory of finite dimensional normed spaces, Lecture Notes in Math., vol. 1200, Springer-Verlag, Berlin and New York, 1986. MR 87m:46038

[Pi] A. Pietsch, Operator ideals, North-Holland, Amsterdam, 1978. MR 81a:47002

[S-T] S. J. Szarek and M. Talagrand, An "isomorphic" version of the Sauer-Shelah lemma and the Banach-Mazur distance to the cube, GAFA Seminar 87-88, Lecture Notes in Math., vol. 1376, Springer-Verlag, Berlin and New York, 1989, 105-112. MR 90h:46034

[Sa] N. Sauer, On the density of families of sets, J. Combin. Theory Ser. A 13 (1972), 145-147. MR 46:7017

[Sh] S. Shelah, A combinatorial problem: stability and order for models and theories in infinitary languages, Pacific J. Math. 41 (1972), 247-261. MR 46:7018

[Sz.1] S. J. Szarek, Spaces with large distance to $l_{\infty}^{n}$ and random matrices, Amer. J. Math. 112 (1990), 899-942. MR 91j:46023

[Sz.2] , On the geometry of the Banach-Mazur compactum, Lecture Notes in Math., vol. 1470, Springer-Verlag, Berlin and New York, 1991, pp. 48-59. MR 93b:46019

[T-J] N. Tomczak-Jaegermann, Banach-Mazur distances and finite dimensional operator ideals, Longman Sci. Tech., Harlow, 1988. MR 90k:46039 44106

Department of Mathematics, Case Western Reserve University, Cleveland, Ohio

Current address: Department of Mathematics, University of Crete, Iraklion, Crete, Greece

E-mail address: deligia@talos.cc.uch.gr 